\title{
Les métiers flous du développement rural
}

Gilles Jeannot, LATTS, Ecole nationale des ponts et chaussées

(Article publié dans : Sociologie du travail, vol 47, $\mathrm{n}^{\circ}$ 1, 2005, p. 17-35.)

Avec la notion de développement tant dans le domaine du rural que dans celui des banlieues populaires ou des politiques de restructuration industrielle, ont émergé des "agents de développement", "chefs de projet", "chargés de mission" en charge de fonctions de "projet" ou "d'animation" incluant à la fois un contact direct avec les «acteurs» du développement et des tâches de coordination de diverses administrations. Les observations ont mis en évidence la singularité de ces positions professionnelles qui tient à l'effacement de tout cadrage. Leurs positions d'emploi sont variées et leurs statuts bricolés : des contractuels de la fonction publique territoriale ou de l'Etat, des fonctionnaires investis d'une fonction secondaire, des agents d'associations ou de bureaux d'études. Au sein de leur organisation il est difficile de leur attribuer un rôle (Bourdieu, 1993 ; Maillard, 2000), ou celui-ci serait défini de manière volontairement contradictoire (Donzelot, Estèbe, 1994); leurs missions sont définies par des injonctions globales (cohésion sociale, développement durable,...) et leurs interventions entremêlées avec celles des élus (Ion, 1990). Leurs parcours, sont particulièrement ouverts (Behar, Estèbe, 1996), incluant des liens forts entre activité militante privée et activité professionnelle (Péraldi, 1995 ; Sipp, Blanc, 2000). Les professions constituées auraient été interpellées (Maillard, 2002 ; Cousin, 1996) mais seraient restées à distance (Ion, 1990), les nouvelles figures ne parvenant pas vraiment à produire, de leur côté, de nouvelles professions (Sipp, Blanc 2000 ; Tapie Grime, 1993). Mais le plus étonnant, et moins souvent souligné, est la persistance de ce phénomène et son extension à d'autres thèmes d'intervention (après la ville, la sécurité et la justice, l'accompagnement à l'insertion, les reconversions industrielles (Pallez, Aggeri, 2002)). D'où le «paradoxe » de ce que l'on ne peut plus appeler des «nouveaux métiers stricto sensu, puisqu'ils ont désormais une vingtaine d'années » (Brévan, Picard, 2000), mais que l'on peine à saisir autrement qu'à travers cette catégorie par défaut du «nouveau », et les questions, d'une part de la spécificité de ces figures professionnelles atypiques et d'autre part des conditions qui permettent qu'elles émergent et durent.

Le cas des agents de développement en milieu rural est alors particulièrement intéressant pour aborder cette question. En effet, ceux-ci ont pu, entre la décentralisation et le renouveau des intercommunalités, concentrer toutes les caractéristiques qui viennent d'être évoquées. Cet exemple est décalé par rapport à la plupart des références précédentes centrées sur le social et la politique de la ville et permet de dissocier la question de ces figures professionnelles de celle de l'évolution 
du travail social. Et surtout c'est l'un des cas les plus anciens; ce qui permet de mettre en perspective ce moment sur une période de plus de trente ans avec des contextes institutionnels variés.

La recherche présentée ici a porté sur les agents de développement rural ayant travaillé dans une région, la Franche-Comté. La fonction «agent de développement rural » ne fait pas l'objet d'une codification. Le critère retenu, proche de la définition la plus partagée dans le milieu, a été de considérer comme tel, un agent (public ou privé) en charge, à temps plein, sur un territoire particulier correspondant aux espaces des actuels Intercommunalités ou Pays, de la mise en ouvre des procédures de développement rural (Plans d'aménagement rural, Chartes intercommunales, programmes européens...) : définition des besoins, collecte des financements, suivi des réalisations ${ }^{1}$. Les premiers agents de développement, selon la définition retenue, sont des contractuels recrutés par les directions départementales de l'agriculture ou les chambres d'agriculture à partir de 1970. Avec la décentralisation, ce sont des structures locales, le plus souvent des associations, qui devront embaucher en CDD de droit privé cela se traduit par une baisse du nombre de postes. L'époque de gloire de ce que l'on appelle alors le «développement local » correspond à une période de basses eaux pour l'emploi. Progressivement, à partir de 1995 et de manière très rapide après 2000, la multiplication des établissements publics de coopération intercommunale (EPCI) et des Pays s'accompagne de nombreuses créations de postes, occupés en grande majorité par des contractuels de la fonction publique territoriale ${ }^{2}$.

\begin{tabular}{|c|c|c|c|c|c|c|c|}
\hline Période & 1970-1974 & 1975-1979 & 1980-1984 & 1985-1989 & 1990-1994 & 1995-1999 & $2000-2003$ \\
\hline Nbre entrées & 12 & 7 & 11 & 8 & 10 & 15 & 32 \\
\hline Nbre postes maxi & 12 & 15 & 19 & 10 & 12 & 23 & 51 \\
\hline Statuts dominants & $\begin{array}{l}\text { Contractuels } \\
\text { Etat + } \\
\text { chambre } \\
\text { agriculture }\end{array}$ & $\begin{array}{c}\text { Contractuels } \\
\text { Etat } \\
+ \\
\text { Département }\end{array}$ & $\begin{array}{c}\text { Contractuels } \\
\text { Etat } \\
+ \\
\text { Département }\end{array}$ & $\begin{array}{c}\text { CDD } \\
\text { Privé } \\
\text { (associations) }\end{array}$ & $\begin{array}{l}\text { CDD } \\
\text { Privé }\end{array}$ & $\begin{array}{c}\text { CDD } \\
\text { Privé } \\
\text { Contractuels } \\
\text { EPCl }\end{array}$ & $\begin{array}{l}\text { Contractuels } \\
\qquad \mathrm{EPCl}\end{array}$ \\
\hline
\end{tabular}

Au cours de ces trois décennies, les actions ont porté sur l'organisation du tourisme,

1110 entretiens ont été réalisés en 2003 (30 avec les plus récemment arrivés par Philippe Chaudat).

Des fiches de parcours ont été établies et exploitées pour 95 agents correspondant à la définition retenue et qui représentent l'essentiel des agents ayant travaillé dans la région. La recherche a été financée par le Centre national de la Fonction Publique Territoriale et par le Ministère de la recherche «CI travail ».

2 Nbre entrées $=$ nombres d'agents occupant pour la première fois un poste d'agents de développement en Franche-Comté sur la période. Nbre maxi poste = nombre maximal de postes simultanément ouverts sur la période. 
la labellisation de produits agricoles, le soutien au petit commerce de proximité, la mise en relation d'entrepreneurs locaux, la création de zones d'activités artisanales, ou des actions d'accompagnement péri-scolaire .... La région se distingue par la faible part des actions liées au développement industriel et par le faible investissement de l'institution régionale qui a laissé la place à des interventions différenciées des Départements.

Le parti a été pris de croiser les approches en visant tous les agents de développement ayant travaillé dans la région depuis les origines : données sur l'emploi, l'activité et les parcours ${ }^{3}$ des agents de développement à partir d'entretiens portant sur l'activité actuelle (commentaire d'agenda), d'entretiens biographiques, de sources écrites. L'analyse des pratiques met en évidence de grandes marges d'autonomie et une capacité d'invention, mais aussi le processus, d'abord lent, puis plus rapide de bureaucratisation (1). Cependant, si on souhaite comprendre la période de flottement de près de vingt ans qui sépare les premières formalisations de la généralisation de la routinisation, et la persistance d'une visée d'efficacité globale et de contact avec la population, l'enquête révèle qu'il faut aller au-delà de l'analyse des pratiques et de leurs codifications bureaucratiques et aborder les conditions sociales de l'emploi (Demazière, Mercier, 2003) et, en l'occurrence, le mode de circulation de l'argent public qui permet le financement de ces postes (2). Durant la période où le financement des postes a reposé sur la captation de subventions se dégage alors la figure des «métiers flous» ${ }^{4}(3)$.

\section{Le travail des agents de développement}

\section{1 L’invention du développement}

Les premiers agents de développement contractuels sont recrutés dès 1970 pour mettre en œuvre les Plans d'aménagements ruraux, procédure qui fait figure d'exercice technocratique. Contrepartie des Schémas directeurs d'aménagement et d'urbanisme 5 , ces plans d'aménagement s'inscrivent dans la logique d'une "solution équipement" : toute intervention se ramenant à la production d'équipements pour répondre à des besoins dûment répertoriés (Jeannot, 2001 b). Ils s'inscrivent aussi dans une époque d'affirmation d'une politique agricole productiviste (Gerbaux, 1998). Les tâches de ceux que l'on appelle alors des «chargés d'études » consistent d'une part à collecter et traiter des données statistiques sur le territoire visé, et, d'autre part, à recueillir des attentes ou demandes formulées par la population, le tout pour définir des «besoins » auxquels répondront des aménagements.

${ }^{3}$ La partie sur les parcours ne sera mobilisée ici que très partiellement.

${ }^{4}$ La notion de «métiers flous » et une bonne part des hypothèses proposées ici ont été élaborées au LATTS avec Viviane Claude que je remercie ici.

5 Institués par la Loi d'orientation foncière de 1967, le décret d'application ne date que de 1970. Les Plans d'aménagement rural s'inscrivent aussi dans la lignée des «Secteurs pilotes d'aménagement rural » introduits par une circulaire agriculture construction du 3 mai 1960. 
Cependant, confrontés directement à la population dans des réunions organisées dans tous les villages, ils vont élaborer les prémisses du développement. Cette expérience est rapportée, par ceux qui l'ont vécue, sur un mode héroïque : énergie nécessaire pour faire plusieurs fois par semaine d'importants trajets en soirée, caractère vierge de milieux socio-économiques qui n'avaient jamais été sollicités de la sorte et grande ouverture des débats qui s'écartaient rapidement des questions d'agriculture et d'aménagement. Le retournement de perspective est particulièrement spectaculaire dans le cas du réinvestissement d'une méthode d'animation de groupes issue de la rationalisation des choix budgétaires (RCB). Cette méthode, qui était alors proposée par les services du ministère de l'agriculture, va être utilisée pour les premiers Plans d'aménagement rural. Elle consiste à développer de manière systématique des chaînes de détermination entre des finalités visées et des actions engagées.

Cela se concrétise, pour le Plan d'aménagement rural de Maiche-Le Russey, par un tableau dans lequel 77 actions (ex. : «adapter l'enseignement technique aux besoins de l'économie locale») sont rapportées à 31 objectifs intermédiaires (ex : « reconvertir partiellement l'industrie horlogère »), puis à 12 objectifs supérieurs (ex : «maintenir une répartition équilibrée de la population ») et à 4 finalités (ex : «avoir un développement de la société équilibré maîtrisé et atteignant 21000 habitants en 1985 »). Les déterminations étant multiples ce sont des centaines de lignes, à l'époque tracées à la main, qui relient toutes les cases qui se déploient sur un double format A3.

Cet outil technocratique, perdu dans une salle de mairie d'un petit bourg rural, ne produira pas les effets attendus. De l'avis de plusieurs agents qui ont utilisé cette méthode dans d'autres territoires, il ne se dégageait pas de priorités claires. En revanche, de ces discussions émergent des transformations importantes: dans le public touché (300 personnes sur 8000 habitants), les solutions proposées (comme une centrale de réservation hôtelière, ce qui était largement sortir de l'origine agricole des promoteurs de la démarche) et les collectifs de travail, en l'occurrence la «commission thématique» qui rassemble élus et acteurs socio-professionnels associés au domaine concerné.

C'est autour de telles commissions thématiques que se jouera ensuite le passage de la programmation dans les Plans d'aménagement rural à une phase de mise en œuvre avec les Contrats de pays en 1975 puis les Chartes intercommunales en 1983. Le tout se passe dans un contexte où les moyens disponibles pour maintenir l'activité dans le milieu rural sont faibles face aux tendances lourdes de désafection de ces territoires liées à l'urbanisation. Une activité tâtonnante de mise en relation du projet de développement et des conditions locales conduit à inventer (ou réinventer) des formes d'intervention publique.

«Tous les équipements qu'on se paye, on les a un peu surdimensionnés pour pouvoir accueillir d'autres entreprises, d'autres activités : ça peut être des associations. On a des contacts avec un cabinet comptable qui serait intéressé d'avoir un bureau là pour mettre ses comptables, mais il n'aura pas de 
secrétariat, pas de téléphone, pas d'ordinateur... nous on lui offre tout cela» (agent de développement ${ }^{6}$ )

Dans cette visée d'économie et d'adaptation se construisent alors de nouveaux partages entre public et privé. A travers cet exemple, où on reconnaît les linéaments de ce que seront par la suite les «pépinières d'entreprises », se dessine un déplacement des partages établis pour l'intervention économique traditionnelle: l'aménagement de zones d'activités. Alors que pour celles-ci, l'autorité publique se contente de proposer aux entreprises des terrains aménagés, les membres de la commission, ici, se mettent à la place de l'entrepreneur pour lui proposer des solutions adaptées. Ce faisant, ils étendent au passage la sphère d'intervention de l'autorité publique vers une offre de services. Cet aspect se retrouve dans la plupart des projets développés au tournant de la décentralisation. Pour le domaine du commerce cela s'est traduit par la mise à disposition de locaux municipaux pour une boulangerie ou d'une camionnette pour une boucherie itinérante, pour la santé par le financement d'un cabinet pour des infirmières et médecins. L'autorité publique intervient aussi directement pour fédérer un secteur économique dans le tourisme (soutien aux projets de gites ruraux et offre d'infrastructures liées au ski de fond), l'agriculture (campagne de promotion pour les vins du Jura ou pour le fromage du Mont d'Or), ou l'artisanat. Dans tous les cas, ces démarches conduisent à rediscuter de manière pragmatique ce qui est vraiment d'intérêt public: la présence d'une boulangerie apparait alors plus essentielle au maintien de la population que la création d'une nouvelle salle communale.

\section{2 La prise en charge du projet et des cofinancements}

Quelques années plus tard, vers le milieu des années $1980^{7}$, la phase d'invention est close, toutefois l'autonomie des agents de développement n'est pas pour autant complètement réduite, ni leur volonté de prendre en charge le développement dans une visée d'efficacité globale. Progressivement les mêmes solutions sont reproduites d'un site à l'autre. Cette uniformisation est renforcée par des procédures de cofinancement. Les procédures thématiques d'Etat ${ }^{8}$ sont maintenues et complétées par des financements issus des fonds structurels européens ${ }^{9}$, mais surtout, c'est par le même mécanisme de distribution conditionnelle de fonds que la Région et le Département construisent leur action territoriale ${ }^{10}$. Le travail se déplace alors de

6 Transcription d'un entretien réalisé en 1988 dans le cadre de l'association nationale du développement local et des pays, cf. infra partie 3.

${ }^{7}$ Les documents sur lesquels repose la partie précédente datent de 1988, ceux-ci portent déjà un regard rétrospectif sur des inventions qui se sont accomplies au tournant de la décentralisation.

8 Opérations programmées d'amélioration de l'habitat (1977), Opérations de restructuration de l'artisanat et du commerce (1994), contrat éducatif local (1998).

${ }^{9}$ En particulier les programmes LEADER (I 1991-94 ; II, 1994-99 ; + (2000-06))

10 «Contrat régional de développement » et selon les Départements : «Plan local de développement », 
l'invention de nouveaux objets d'interventions vers l'effort pour faire émerger une action cohérente à partir de multiples composantes normées. Il porte alors d'une part sur la mobilisation des différents « acteurs » du développement autour d'un «projet» et d'autre part sur l'ingénierie des cofinancements.

Si les solutions proposées s'inscrivent dans un répertoire bien défini, l'enrôlement des «acteurs» nécessite toujours un «travail politique » (Terssac, 2003). Par-delà l'enthousiasme et la force de conviction, ils s'agit de trouver des manières de compenser divers désajustements entre ceux-ci. Cela inclut par exemple de faire évoluer les découpages de territoires : ainsi un agent de développement a pu prétexter que la zone couverte par son association de développement était trop étroite pour une procédure (en l'occurrence une Opération programmée d'amélioration de l'habitat) pour engager une collaboration avec une petite ville voisine, le nouvel espace de référence préfigurant le Pays à construire.Cela peut aussi conduire à agir de manière différenciée selon les interlocuteurs: par exemple concilier des besoins de transparence de la démarche participative et le minimum de discrétion qu'attend un investisseur privé. Cela inclut aussi la prise en compte de rythmes différents, la maturation d'une idée dans un milieu associatif devant se faire avant d'avoir trouvé les interlocuteurs politiques ${ }^{11}$.

Une fois la mobilisation sociale engagée et les orientations choisies, la seconde composante du travail consiste à mettre les différentes sources de co-financement au service de cette construction fragile. Ceci est rendu possible par le fait que paradoxalement, la multiplication de ces codages de l'activité, du fait de leur enchevêtrement et de leurs inévitables décalages, contrebalance les effets d'encadrement propres à chacun d'eux. Si, vu d'en haut, chaque aide est conditionnée à des critères affichés et scrupuleusement vérifiés, vu d'en bas, les différences d'orientations entres ces différents financeurs, laissent d'importantes marges d'action locale. Il est en effet toujours possible de découper un projet en de multiples opérations et d'affecter chacune au financeur le plus sensible. Une telle pratique a même été institutionnalisée au niveau départemental, dans le Jura, vers 1990, autour d'un groupe technique de professionnels qui rassemblait à la fois des cadres de l'Etat, du Département et les agents de développement. Dans ce groupe, les différents projets étaient présentés et discutés et les plans de financement constitués. Les crédits qui comportaient le moins de contraintes de contenu étaient alors affectés aux projets qui ne répondaient pas aux critères des autres financements. Plus tard, dans un contexte où une telle connivence entre techniciens de l'Etat, du Département et des Intercommunalités ne sera plus de mise, le partage pourra encore se jouer au niveau de chaque territoire comme l'indique l'affectation conforme aux critères de chaque financeurs des composantes d'un programme touristique de l'association des développement et d'aménagement du Val d'Amour (Jura) en 1995 (Vaivre, 2001) ${ }^{12}$ :

contrat « APPUI » ou « FIDEL ».

${ }^{11}$ Ce travail de mise en relation est décrit de manière plus détaillée dans (Jeannot, 2001 a) ou dans (Nay, Smith, 2002).

12 Les actions inscrites dans plusieurs cadres sont co-financées. 


\section{Insérer figure cofinancements}

Toute la culture professionnelle, exprimée tant dans les collectifs qui prennent forme que dans les formations dispensées, tourne alors autour de cet effort pour, en les maîtrisant complètement, faire fi des contraintes de financement, et passer du « guichet au projet ».

\subsection{La bureaucratie des contrats}

Cependant, aujourd'hui, les agents de développement récemment recrutés et qui ont suivi pour la plupart une formation au «projet» reconnaissent à regret le retournement régressif $\mathrm{du}$ «projet au guichet ${ }^{13}$. Ils font le constat amer qu'aujourd'hui la machine tourne à vide. Tous les agents actuellement en poste évoquent l'activité rhétorique "d'habillage" des projets destinée à les faire rentrer dans les critères affichés. Ils ont appris à être maîtres des termes inscrits dans les documents d'orientation des financeurs ${ }^{14}$.

“Par exemple, aujourd'hui, on sait très bien que pour faire passer un dossier sur les crèches-halte garderies, pour que ça soit financé par l'Europe, il faut dire que notre préoccupation principale c'est qu'on veut améliorer l'égalité des chances entre les hommes et les femmes. Et le fait de créer une crèche avec, pourquoi pas, des horaires atypiques d'ouverture le soir ou entre midi et deux, ça permettrait aux femmes de prendre des responsabilités dans des associations, d'être élues dans une commune ou de pouvoir travailler plus tard dans leur vie professionnelle et d'avoir de meilleures responsabilités, sinon, si on fait une crèche juste pour faire une crèche, c'est pas financé » (agent de développement).

La plus value de l'agent de développement se réduit alors à être régulièrement en contact avec les agents qui instruisent les dossiers, certains insistent même sur la nécessité de se rendre dans leur bureau et de les connaître personnellement. On retrouve aussi la même réduction dans les formes d'ouverture à la population. Les Conseils de développement des pays, censés perpétuer l'esprit d'ouverture des premières associations, sont, pour la plupart, dans la région étudiée, largement

13 Ce constat qui est apparu dans de nombreux entretiens avec les nouveaux arrivés a été confirmé dans une séance de restitution de l'enquête devant un public composé d'agents de toutes les périodes.

14 Comme le DOCUP pour les financements européens. 
cooptés par les élus.

La comparaison pour les Intercommunalités et Pays, des activités actuelles des agents anciens et de ceux pour lesquels c'est le premier poste conduit d'abord à nuancer mais ensuite, au fond, à renforcer cette analyse. En effet, il y a bien une différence sensible des pratiques. L'effet de contraste est renforcé pour ceux qui sont depuis longtemps sur un même territoire dont les «acteurs » ont acquis une expérience et qui sont soutenus par le même élu entrepreneur. Les contre-exemples à l'analyse précédente sont le fait d'agents plus anciens : création de formes innovantes d'intervention, situations dans lesquelles la définition de l'action précède la recherche de financements, relations suivies avec des milieux économiques (tournée d'enquête auprès de toutes les entreprises, animation d'un club d'entreprises d'une même filière) et associatifs (obtention d'un programme LEADER avec des associations culturelles). Mais dans le même temps ceux-ci expriment les difficultés à maintenir ces manières de faire.

Le processus décrit suit donc un mouvement classique de passage progressif de l'innovation à une routinisation. Mais comment expliquer la longue période durant laquelle la part d'autonomie des agents reste très importante, et le fait que les agents, dans le but de donner un sens au projet global, tirent alors parti des contradictions liées à la multiplicité des financements pour ne plus le faire ensuite ? Il faut pour cela prendre en compte une autre modalité d'institutionnalisation qui ne passe plus par des procédures et des codifications mais par une redistribution des rôles.

\section{Sources de financement des emplois et relation aux élus}

La question de l'autonomie des agents de développement ne peut être abordée à partir de la seule analyse des pratiques et suppose aussi une prise en compte des conditions sociales d'organisation du travail, second thème de l'enquête. Un poste de cadre, même relativement mal payé, représente un budget substantiel rapporté aux investissements publics dont il est question ici. Dans tous les cas les salaires des agents de développement sont issus de fonds publics et in fine de l'argent des impôts. Mais le circuit qui va du contribuable à la fiche de paie de l'agent joue un rôle ; les relations entre ces agents et les élus locaux ne sont pas les mêmes dans le cas d'un passage direct pour le financement d'un poste d'agent d'une collectivité territoriale ou indirect via des politiques de subvention.

\section{1. Des contractuels de l'Etat au service du territoire}

Les premiers agents de développement sont financés par l'Etat. Leur recrutement pour la mise en œuvre des Plans d'aménagement rural dépend de l'initiative des ingénieurs fonctionnaires des Directions départementales de l'agriculture (DDA), comme en témoigne le contraste entre les différentes DDA de la région étudiée ; alors que la DDA de la Haute Saône recrutera sept agents avant la décentralisation, un seul le sera dans le Doubs. Une fois recrutés et affectés à un territoire, ces agents contractuels de l'Etat ou du département se trouvent mis « gratuitement » au service d'un espace découpé selon des critères techniques, qui préfigurent ce que seront vingt 
ans après les «Pays» des lois Pasqua et Voynet mais qui ne sont pas encore constitués en entités politiques. Libres de toute relation de dépendance vis-à-vis des élus locaux, ils vont pouvoir établir des liens avec les interlocuteurs qui leur paraissent les plus dynamiques et les plus représentatifs de la société locale. Leur relation à la société politique locale est alors démiurgique : ils ont pour tâche de la faire émerger. L'image qui s'impose, en forçant le trait, est celle d'équipes commando qui débarquent sur un territoire et bousculent des ordres établis en donnant la parole à des acteurs locaux non élus, plus proche en cela de la tradition du Plan que des arrangements avec les notables locaux (Grémion, 1976).

Les réunions d'information dans les villages permettent de repérer des «acteurs » locaux et de les inciter à venir par la suite participer à des commissions thématiques (agriculture, commerce, tourisme, ....) correspondant à leurs préoccupations. Les élus ne sont pas alors majoritaires dans les commissions (Roch, 1983). Ensuite, le mot d'ordre est de «créer une association » avec le même mélange de dirigisme technocratique et d'ouverture démocratique que dans le cas du réinvestissement de la RCB évoqué précédemment.

«On appliquait à chaque nouveau territoire un canevas type de statut avec un certain nombre de postes au conseil d'administration pour représenter les élus, un certain nombre pour représenter les socioprofessionnels, un certain nombre pour représenter les associations. Ici les gens disaient "le PAR" (plan d'aménagement rural) pour désigner l'association. On continuait de dire "le PAR" mais l'association avait pris son autonomie. » (agent de développement).

Des formations sont organisées pour les élus sur des sujets comme les finances communales. Elles seront aussi ouvertes à des non élus qui ont été sensibilisés à ces questions dans les commissions de travail des Plans d'aménagement rural. Plusieurs agents notent alors que ces non-élus constitueront le vivier de renouvellement du personnel politique local :

"Cela a engagé une certaine dynamique de renouvellement des élus à l'époque, je ne veux pas dire que c'est nous qui avons fait changer les élus, mais je veux dire qu'il y a un certain nombre de gens qui sont venus à ces réunions là, qu'on a réussi à former, cela leur a permis de comprendre le fonctionnement d'une société locale, comment cela se gérait et qui ont pris goût pour certains et il y a en un certain nombre qui sont devenus des maires, certain y sont encore." (agent de développement).

Cette dynamique s'est alors déployée au-delà des territoires de chaque plan. Les acteurs locaux qui ont été mobilisés autour de l'idée de «pays» ou de «petite région » vont envisager de trouver des relais entre eux. Ainsi en 1978, la plupart des agents rencontrés étaient-ils tendus vers l'organisation d'une rencontre aux salines d'Arc et Senans de toutes les expériences de «pays ». Le caractère déstabilisateur de l'ordre politique local, d'une telle réunion n'a pas échappé au préfet de région qui interdira sa tenue deux jours avant la date prévue.

\section{2. Des couples agent de développement / élus}

Cette modalité d'intervention descendante et portée par l'initiative de fonctionnaires d'Etat ne durera pas longtemps après la décentralisation. En Haute Saône, certains 
agents qui avaient négocié des contrats à durée déterminée longs associés au suivi d'un Plan d'aménagement rural pourront continuer à occuper leur poste deux ou trois ans. Il en va de même, dans le Jura, d'agents qui auront obtenu, juste à cette période, un statut relativement protecteur d'agent de développement départemental, l'un d'eux est encore en poste. Et dans le Doubs, l'agent de la Direction départementale de l'agriculture poursuivra son activité dans le cadre d'une mise à disposition des services du Département.

Une autre mode de financement, et un autre type de posture de l'agent de développement vont devoir être trouvés. Il avait déjà été expérimenté avant la décentralisation sur plusieurs territoires dans le Jura et dans le Territoire de Belfort. Sur ces sites, les agents devaient se "créer" leur poste en cumulant des ressources issues d'une cotisation des communes, de subventions du Conseil général, mais aussi de prestations associées à la mise en oeuvre de procédures ou effectuées pour le compte des communes. Dans ces conditions, les emplois n'existent que là où une volonté locale s'est constituée et en particulier là où des élus ruraux volontaires, ayant pour la plupart des visées politiques dépassant leur territoire, vont faire le pari de l'intercommunalité mais aussi le constat que celle-ci ne peut plus se déployer sans un agent de développement. De ce fait, ils s'attachent alors, l'élu pour se dessiner une envergure politique ${ }^{15}$, l'agent pour financer son emploi, à capter des subventions émanant de l'Etat et du Département puis de la Région et de l'Europe. Ils sont alors interdépendants, le poste d'agent de développement ne peut exister sans une volonté politique, mais en retour il permet à l'élu de mobiliser des ressources qu'il n'aurait pu obtenir seul ${ }^{16}$.

L'image d'un agent de développement prenant la place de l'élu, bien que répandue chez les nouveaux venus, ne correspond pas à la réalité évoquée ici. Il est remarquable de ce point de vue que l'agent qui est le plus souvent cité comme "l'agent de développement typique" par sa capacité d'auto définition de son rôle soit, du point de vue du statut, dans la position exceptionnelle d'avoir négocié avant la décentralisation un contrat en CDI avec le Département. Hors de ces conditions particulières, un agent de développement fort face à un milieu politique faible, "politique à la place du politique", n'est pas vraiment possible. La figure la plus répandue est plutôt celle d'un élu qui sur le terrain fait couple avec son agent de

15 En l'occurrence : deux sénateurs-maires, deux conseillers généraux qui sont devenus présidents du conseil général, un ex agent de développement devenu conseiller général et un conseiller général qui a échoué à la députation

16 Alain Faure (1992) a indiqué comment des maires ruraux entrepreneurs ont appris à cette époque à dénicher des subventions pour leur commune. Les élus évoqués ici ont des ambitions plus larges que leur commune et ont besoin d'une ingénierie pour constituer les dossiers et suivre les opérations. Contrairement au couple élu /secrétaire général (Burlen, 1998), la dépendance est double ; l'agent est recruté par l'élu mais le projet de l'élu dépend aussi des ressources mobilisées par l'agent de développement. 
développement, le projet de développement étant pensé de manière bicéphale et mis en oeuvre à deux. Leurs interventions se composent de manière intriquée, le politique rentrant dans les dossiers techniques et l'agent de développement portant le projet politique.

Même lorsque le développement se construit autour d'une association d'agriculteurs et non d'un élu, on retrouve un agencement équivalent entre un leader d'association et un agent de développement. Dans deux des trois territoires concernés dans la région, c'est même de l'activisme d'agents et d'administrateurs bénévoles d'un bureau d'études que sont nées les associations. Le besoin d'un complément entre mobilisation sociale et équilibre des financements s'exprime alors encore plus clairement.

« En 1991 il y avait deux grandes zones objectifs 5b avec le Jura qui marchait bien et le nord de Haute Saône qui ne marchait pas bien. Ce secteur ne marchait pas bien car il n'y avait pas d'initiatives locales pour aller chercher les projets. Il y avait eu des chantiers internationaux qui avaient permis à des acteurs locaux de se dire qu'il faudrait qu'on arrive à susciter une dynamique. (Notre bureau d'études) a été sollicité car il y avait des administrateurs de là bas. J'ai été recruté sur cette mission là en 1991." "La logique d'un organisme comme (le nôtre) c'est d'investir à perte, et ensuite, quand le territoire s'est organisé et qu'il décroche un programme leader II, c'est vrai qu'il doit y avoir un retour sur investissement, l'organisme va intervenir, là, sur des opérations financées. Par ailleurs nous, dans l'attente d'avoir cela, on était sollicité sur des programme d'insertion cela permettait d'avoir une action de développement et d'être financés. ». (agent de développement, bureau d'études).

Cette posture singulière place le couple agent de développement / élu (ou leader d'association) dans la position d'entrepreneurs qui doivent simultanément produire la légitimité et le financement de leur action. En effet, pour faire tenir une couche supplémentaire de l'action publique au-dessus des communes et des maires qui ont la légitimité élective, ils doivent à la fois montrer qu'ils répondent aux attentes de la population et que leur intervention est largement financée par des ressources externes. La quête d'une validation de l'action dans la société civile ne cesse alors d'être présente. Ceci permet d'expliquer la répétition, pour des opérations comparables sur le même territoire, de «diagnostics » qui incluent traitement de données statistiques, et enquête auprès de la population.

La forme juridique majoritaire des employeurs, qui correspond à cette forme de financement de l'action, est l'association ; c'est le cas pour les associations issues du milieu agricole, pour les bureaux d'études issus de mouvements sociaux mais aussi pour les organismes soutenus par des élus locaux. Dans ce dernier cas, les agents de développement sont alors employés d'une association de développement laquelle effectue des prestations pour le compte d'un syndicat de développement. Ce mode d'organisation a perduré malgré les critiques de "gestion de fait" qui ont été avancées par plusieurs préfets successifs. Les emplois sont souvent faits de bricolages et d'arrangements divers. Ainsi, à titre d'exemple, un agent est employé par la fédération des œuvres laïques pour pouvoir bénéficier d'un crédit de soutien à l'animation alors qu'elle travaille de fait pour une intercommunalité. Le salaire est lui aussi le plus souvent issu de sources multiples. 


\subsection{Les agents de développement des Pays et nouvelles Intercommunalités}

Les conditions créées par la montée de l'intercommunalité et des pays transforment les données du financement des agents de développement. Même si l'évolution est progressive, le financement par l'impôt (taxes locales et DGF) affecte les relations entre élus et agents de développement et même avant cela entre le président de l'intercommunalité et les maires des communes. Comme le note une femme agent de développement présente sur le même territoire depuis 1982 :

«Ce qui a changé le plus (avec la Communauté de commune), je pense, c'est la référence à un groupe d'élus, il y a un bureau qui se réunit régulièrement, il y a plus de compte rendu aux élus responsables qu'il n'y a en avait. Les élus de la communauté sont plus soucieux et plus présents que ne l'étaient ceux de l'association. » (agent de développement).

Il ne s'agit plus de construire simultanément la légitimité d'un élu et le financement d'un poste en captant des subventions qui tombent du ciel mais d'offrir des prestations à des communes et à travers elles à des contribuables, électeurs par ailleurs.

Cela se traduit dans les formes organisationnelles. Au modèle de l'extension par cellules (un agent de développement généraliste responsable et des «chargés de mission » recrutés pour la mise en œuvre d'une procédure et dont le contrat s'achève avec celle-ci) se substitue progressivement un modèle administratif plus classique. Le titre "d'agent de développement" est alors attribué au responsable du service économique, qui exerce sous la direction d'un secrétaire général (issu de la fonction publique territoriale). Il participe alors surtout à la production et à la gestion de zones d'activités économiques plus proche des démarches d'aménagement que des recompositions du développement. Et lorsque c'est le responsable du service qui garde le titre d'agent de développement, ses tâches de gestion administrative deviennent envahissantes et il reconnaît alors devenir autant secrétaire général de l'intercommunalité qu'agent de développement. Les responsables des nouveaux Pays qui ont été créés sur un statut d'association marquent aussi leur volonté d'institutionnalisation en en faisant des établissements publics.

Corrélativement, les relations avec les élus se transforment notablement. Au projet partagé, fait place la recherche, proche de ce que l'on observe dans les collectivités locales, du respect des rôles. Ils cherchent alors, comme de nombreux fonctionnaires territoriaux (Lorrain, 1989; Burlen, 1998) à trouver la bonne distance entre intérêt du travail et force de proposition d'une part et respect des prérogatives des élus d'autre part: savoir ne pas se mettre en avant dans une assemblée publique, suggérer plus que proposer, ...

La rapidité du changement, qui est telle que l'on pourrait presque penser qu'il y a rupture de continuité et que le même terme, «agent de développement » recouvre des fonctions sans relations, met en évidence le poids du cadre institutionnel sur les pratiques. 


\section{Des métiers flous?}

$\mathrm{Au}$ croisement de ces deux lectures, sur le travail et sur les conditions d'emploi, la position des agents de la période intermédiaire apparaît singulière. Cette période peut être délimitée assez précisément si on se réfère au contexte institutionnel. Elle débute quelques années après la décentralisation, lorsque s'éteignent les contrats à durée déterminée financés par l'Etat et le Département, soit vers 1985. Elle se clôt avec l'émergence des intercommunalités, le mouvement est entamé à partir de 1995, mais il se traduit de manière très forte à partir de 1999 avec une croissance rapide du nombre de postes ${ }^{17}$. Si on se place du point de vue des individus, et de leur date d'entrée, le partage ne peut être aussi tranché. D'une part, des personnes entrées avant la décentralisation, à partir de 1980, se sont trouvées mises sur le marché et ont participé activement à cette deuxième phase, et d'autre part les anciens agents de développement encore en poste, même si le poids du contexte est réel, emportent avec eux une part des orientations de cette période intermédiaire.

Une partie des agents qui ont exercé durant cette période revendique l'appartenance à un «métier» qui ne semble, cependant, que partiellement se constituer. C'est le terme qui s'impose pour les regroupements professionnel au niveau national. Une des organisations les plus revendicatrice a pris pour titre «Agent de développement : un métier à défendre », le réseau des associations dans le domaine s'appelle «Plateforme des métiers », et propose entre autres de regrouper les «référentiels métier » d'agent de développement pour en faire une synthèse. L'usage de ce terme est relié à la constitution d'un collectif ayant prétention à un contrôle sur l'accès aux emplois et sur le contenu des interventions, une visée de profession (Dubar, Tripier, 1998), et ne peut être considéré comme une simple «sténographie pratique » (Sewel, 1983) détachée de la référence aux corporations. Il fait aussi écho à des formes plus contemporaines du «désir de métier»(Osty, 2003) : l'attachement à un métier comme «patrimoine » garantissant l'individu en cas de mobilité mise en avant par Françoise Piotet (2002), et l'attente de reconnaissance d'un espace d'autonomie dans le travail pointée par Florence Osty (2003). Toutefois, si ces trois dimensions (collectif, patrimoine, autonomie) sont bien présentes en partie et permettent de reprendre le terme de «métier », celui-ci ne se laisse pas complètement délimiter.

En premier lieu, il y a eu plusieurs tentatives de fédération des agents de développement dans la région, mais la mobilisation collective n'apparaît pas dissociable du mouvement de promotion du développement. Le premier regroupement professionnel apparaît en 1988 dans le cadre de «l'association nationale du développement local et des pays ». En 1991 est créée une «Entente pour le développement global en région Franche Comté », et en 1998 ADELE Franche Comté, en liaison, cette fois, avec l'UNADEL association nationale de promotion du développement. Cependant la constitution de ces associations reflète la difficulté de séparer le projet politique du développement et l'organisation professionnelle des agents. La principale activité du premier regroupement a été de faire conduire par des

\footnotetext{
179 agents entrés en 1999.
} 
agents de développement des entretiens auprès des principaux élus innovateurs dans ce domaine. Les statuts de la seconde ont été rédigés par un agent de développement devenu conseiller général et son siège était au Conseil Régional ${ }^{18}$. Et la troisième comme la seconde sont d'abord des associations de structures de développement, même si elles abordent la question du «statut des métiers du développement ».

En second lieu, les parcours accomplis ne traduisent que partiellement la constitution d' « une compétence décontextualisée » (Piotet, 2002), un patrimoine reconnu par les pairs qui aurait une valeur sur le marché du travail. Deux groupes d'agents mettent en avant deux conceptions du métier liées à leurs parcours et les font valoir dans des associations professionnelles. Le premier groupe est constitué par ceux qui ont occupé plusieurs postes d'agents de développement dans des territoires différents. Ils valorisent une décontextualisation liée à la différence géographique et à la diversité des contextes politiques. Les agents du second groupe, qui ont eu une expérience dans un bureau d'études, proposent une définition de la compétence plus large fondée sur la mise en avant de savoir faire transversaux, comme la réalisation de diagnostics et la conduite de projets, et valorisent des perspectives de mobilité vers d'autres domaines du développement. Cependant ces conceptions du métier apparaissent fragiles lorsqu'on les rapproche des parcours effectifs de ceux qui les portent. Si on approfondit les parcours des agents du premier groupe on note que, dans la majorité des cas, la mobilité s'explique rarement sans des difficultés liées à une trop grande proximité avec l'élu de référence, ce qui relativise la prétention à des compétences purement techniques. Les parcours des agents du second groupe sont à la fois marqués par l'engagement militant (les deux principaux bureaux d'études de la région dont issu de mouvements syndicaux) et par une très grande variété. Ceux-ci évoquent spontanément leur caractère atypique ${ }^{19}$.

En troisième lieu, la dimension du métier mise en avant par Florence Osty (2003) («forme d'activité spécifique marquée par la maîtrise d'un espace d'autonomie » et qui traduit une « visée de l'oeuvre ») correspond, elle, tout à fait, mais peut-être trop, aux agents de développement, même lorsqu'ils ne sont pas mobiles. La manière dont ils s'engagent conjointement avec leur élus ou leader d'association dans la constitution d'un projet déborde la sphère de simple autonomie dans l'exécution. Et les agents nouvellement arrivés ne manquent pas de dénoncer le risque de confusion des genres associé à cette trop grande autonomie. La question de l'imprécision des frontières avec le politique obère donc encore la capacité de fonder solidement une spécificité professionnelle sur cet espace d'autonomie. Ainsi, dans les trois

18 Son acronyme, «EDGAR », aussi, est transparent pour qui connaît le rôle d'Edgar Faure dans la Région.

19 Un autre argument limitant est lié au fait que pour les agents entrés entre 1980 et 1998, un tiers seulement participe de cette décontextualisation (respectivement 5 sur 35 et 7 sur 35), le reste est constitué d'individus restés agent de développement sur le même territoire (11 sur 35) pour lesquels la décontextualisation ne joue pas ou d'agents ayant fait carrière dans les administrations (Etat et collectivités locales) (10 sur 35) pour lesquels c'est plus la connaissance d'un domaine de l'action publique qu'un «métier» qui a été leur patrimoine. Seuls deux individus sont passés dans des secteurs totalement étrangers au développement rural 
dimensions évoquées, la référence au «métier » n’offre pas vraiment de repères clairs et tangibles, ce sont encore des contours imprécis qui se donnent à voir : les métiers sont flous ${ }^{20}$.

Cette difficulté à délimiter un «métier » n'est que la touche finale de toute une série d'indéfinitions ou d'ambiguïtés qui permettent de rapprocher le cas des agents de développement de celui d'autres positions professionnelles évoquées dans l'introduction. Cela concerne trois domaines : les objets visés et les compétences nécessaires pour les appréhender, les organismes employeurs et les rôles qu'ils confèrent et enfin, plus directement attaché à la position des individus, les catégories de l'emploi et des carrières. Sinon une impossible «définition précise du flou », se dégage alors une cartographie des composantes de la constellation dont les éléments assemblés donnent cette impression généralisée de manque de netteté et de cadrage.

L'objet d'intervention est parfois à peine suggéré par des prescriptions ouvertes sous forme de slogan. Au «développement local» des agents étudiés font écho les injonctions à «l'économie solidaire », ou à « la proximité » invoquée aussi bien pour l'action municipales les fonctions régaliennes de sécurité et de justice que pour les services publics qui ont créé de nouvelles formes de projection territoriale. Souvent, les objets visés sont composites ${ }^{21}$ et ne permettent pas de fixer la focale sur une seule technicité. Ce qui vaut pour le développement à la jonction du politique et de l'économique, se retrouve dans l'environnement avec des objets abordés simultanément dans leurs dimensions scientifiques et juridiques (Lascoumes, Le Bourhis, 1997) ou dans le secteur de l'insertion par l'économique lorsque les acteurs ne se contentent pas d'une action sociale sur les personnes exclues du marché de l'emploi mais prétendent simultanément transformer les conditions d'accueil au sein des entreprises (Wuhl, 1996). Concomitamment, comme on l'a vu à propos du savoir faire en matière de co-financements, l'intervention suppose de savoir composer avec des partenaires administratifs multiples. L'activité se construit alors autour d'une posture et de savoir faire d'entre-deux. C'est la dimension qui a le plus retenu l'attention des observateurs qui ont multiplié les dénominations : «traducteurs » ou «généralistes » (Nay, Smith, 2002) pour mettre en avant le travail cognitif de mise en équivalence de catégories hétérogènes ou pour ne pas négliger le fait que les agents doivent avoir un minimum de technicité dans chacun des domaines qu'ils abordent, « intermédiaires» (Baron et alii, 1994), «animateurs» Donzelot (1994) «médiateurs » (Gaudin, 1999) pour désigner l'activité de mise en relation de différentes parties. Saisies à travers ces grandes fonctionalités, les compétences mobilisées semblent enfin difficile à caractériser plus précisément et à hiérarchiser. Il apparaît par exemple délicat de différencier les compétences génériques des agents de développement (fortement qualifiés) de celles des «médiateurs » dans les espaces

\footnotetext{
20 Le pluriel est employé car plusieurs approches du métier d'agent de développement sont en concurrence.

${ }^{21}$ D'autres manières de caractériser ces objets sont possibles, par exemple autour d'un usage extensif de la notion de risque (Padioleau, 1999), ou de territoire, ce qui importe ici c'est qu'ils ne sont pas saisissables sans un travail.
} 
publics qui ont été recrutés sur statut d'emploi jeunes (faiblement qualifiés) (Sipp, Blanc, 2000$)^{22}$.

Ils sont employés par des organismes aux marges des administrations de l'Etat et des collectivités territoriales. Le cas des agents de développement rural dans les années 1985-1995 qui ont occupé l'espace non encore institutionnalisé de l'intercommunalité, dans des structures associatives, peut être rapprochée dans cette perspective institutionnelle de celui des premiers «chefs de projets » de la politique de la ville, au centre d'un système de financement multipartite et dont le poste était cofinancé par l'Etat et les Communes, avant qu'ils ne soient progressivement «municipalisés ${ }^{23}$. Le décalage peut prendre la forme d'une «mission» hors organigramme, d'une annexe (comme les «maisons » de justice, de service public,..), cela peut être un organisme satellite des collectivités locales (une société d'économie mixte (Pallez Aggeri), ou une association de développement dépendant complètement des fonds publics. Mais cela peut être des organismes plus autonomes comme des bureaux d'études, des associations fondées sur un mouvement social ou des associations qui fonctionnent comme de petites entreprises offrant des prestations aux pouvoirs publics (entreprises d'insertion, organismes oeuvrant dans le social ou la santé publique). De tels contextes institutionnels contribuent à l'ambiguïté des rôles, peutêtre source de souffrance (Bourdieu, 1993), mais aussi générateur d'action. Comme on l'a vu pour les agents de développement, la quête d'une légitimité pour l'association a contribué a maintenir l'effort d'ouverture vers la population et les potentiels entrepreneur; a clarification des rôles avec la création des établissements publics de coopération intercommunale est allé de pair avec l'abandon de cet effort.

Ces organismes para-administratifs n'offrent,enfin, souvent que des emplois précaires ce qui déstabilise les individus. Cet aspect a été moins discuté que les précédents. Pourtant l'exemple des agents de développement rural met en évidence les effets de cette précarité. Sur les entrées d'une part, avec une baisse du niveau scolaire des recrutés ${ }^{24}$ pour cette période pour des emplois peu attractifs et aussi l'arrivée d'individus qui ont connu des parcours variés (élevage de chèvre, tissage, syndicalisme agricole, ...). Mais aussi sur les sorties, avec la remise sur le marché d'une partie des agents qui devront s'inventer un parcours qui valorise cette expérience dans d'autres contextes. L'absence d'un statut d'emploi stable, pour une partie des agents, favorise ainsi la porosité de la catégorie et l'extension de l'espace des parcours, avec une ouverture vers le secteur privé (bureaux d'étude) ou comme cela a été souligné pour la politique de la ville (Péraldi, 1995) vers des parcours militants ou des carrières politiques. Ceux qui ont les parcours les plus chahutés

\footnotetext{
${ }^{22}$ La question de l'extension de la notion de «métiers flous » à cette dernière catégorie reste à discuter.

23 Pour Philipe Mouton (1995) au début des années 1990, $20 \%$ des chefs de projet sont des fonctionnaires territoriaux, $30 \%$ sont employés par les villes en tant que contractuels, 20 à $25 \%$ par une association, $10 \%$ appartiennent à un bureau d'études, $5 \%$ sont employés par une société d'économie mixte ou un organisme HLM et $7 \%$ mis à disposition par d'autres organismes.

${ }^{24}$ Sur les 35 agents entrés entre 1980 et 1998, 8 ont un niveau de formation initiale IV ou inférieur et 7 un niveau II contre respectivement 1 et 6 pour les agents entrés entre 1999 et 2003 et 1 et 1 pour les 19 agents entrés entre 1970 et 1979.
} 
doivent déployer un effort pour réordonner l'ensemble de leurs expériences, ce qui implique une forte «exposition de la personne » (Ion, Péroni, 1997) dans le travail et une plus faible séparation de la sphère professionnelle: «la dimension professionnelle est indissociable de la dimension personnelle » (Béhar, Estèbe, 1996). Tout ceci, comme on vient de le voir, sans que le modèle professionnel du «métier » ne permette de ressaisir et de réordonner ces parcours ouverts en offrant une alternative aux modèles des carrières de la fonction publique.

Ainsi, par métonomymie, on peut étendre du contenant au contenu l'expression «métiers flous» pour recouvrir l'ensemble des caractéristiques des ces figures professionnelles. Plus qu'à l'une ou l'autre de ces composantes, c'est à leur composition que l'on peut associer cette expression. Il n'est alors pas étonnant que cette accumulation rejaillisse alors sur l'identité externe des agents et sur le métier au sens commun d'activité reconnue ${ }^{25}$ par la société. A la question «Lorsqu'au cours d'un mariage ou d'une fête de famille, une tante ou un cousin éloigné vous demande ce que vous faites dans la vie... », la première réaction, pour les agents de cette période, aussi spontanée que partagée, est un rire généreux.

\section{Conclusion}

Les agents de développement rural entrés dans le métier entre 1980 et 1998 cumulent les caractéristiques évoquées en introduction : missions définies de manière vague, positions d'emplois bricolées et parcours influencés par la sphère politique. Si certains aspirent à constituer et à s'inscrire dans un métier, et si un certains nombre des caractéristiques correspondantes sont bien présentes, leurs limites ne sont jamais clairement établies, ce qui justifie l'emploi de l'expression «métiers flous ${ }^{26}$.

La présentation retenue a mis l'accent sur le rôle des conditions de financement des emplois et des relations de dépendance avec les élus qu'elles génèrent. En ce sens on pourrait dire que les «métiers flous » du développement rural sont une conséquence de la manière dont le contrat a été «la mise en œuvre de la décentralisation» (Gaudin, 1999). Les procédures de financement ouvrent l'opportunité de la formation d'un couple entre un élu qui investit l'espace encore non institutionnalisé de l'intercommunalité et un agent de développement qui finance son poste. En outre, agents de développement et procédures contractuelles semblent en symbiose. C'est de la multiplication et des contradictions entre celles-ci que naissent les marges d'autonomie, mais c'est aussi parce qu'ils réordonnent au niveau local les diverses opportunités offertes qu'une action efficace est envisageable dans cette cacophonie d'incitations financières. L'étonnante durée du phénomène, point de départ de l'interrogation, le fait que la situation observée et ces agents aux «métiers flous » se maintiennent plus de quinze ans, ne peut être dissocié de ces conditions

\footnotetext{
${ }^{25}$ Cet aspect du métier est présent dans deux des définitions du Robert.

${ }^{26}$ Le pluriel a été retenu car, comme on l'a vu, plusieurs définitions concurrentes du métier sont présentes.
} 
institutionnelles. Cette lecture est renforcée par le constat de la rapide recomposition qui a suivi la mise en œuvre de la loi Chevènement.

Cependant, si on porte l'attention en amont, une autre lecture, complémentaire, se dessine. En effet, avant toute recomposition institutionnelle liée à la décentralisation, et avec des agents à la fois fonctionnaires pour l'initiative et contractuels à statut stable pour la mise en œuvre, plusieurs caractéristiques de cette situation singulière sont déjà présentes. Ces transformations sont associées à une évolution des objets de l'action publique et en l'occurrence au passage «de la gestion de la croissance au développement du territoire » (Duran, Thoenig, 1996) et corrélativement à un élargissement des acteurs impliqués : associations, investisseurs privés. Dans cette perspective, les «métiers flous» peuvent êtres saisis, au même niveau que les procédures contractuelles, comme symptômes conjoints d'une même évolution de fond de l'action publique. Une telle lecture permet alors de faire le lien avec d'autres positions professionnelles qui apparaissent, elles, bien après la décentralisation et qui ont été associées à d'autres objets, comme les reconversions industrielles, la sécurité urbaine ou le renouveau de la démocratie participative municipale, sans toujours coïncider avec les conditions institutionnelles analysées ici. Dans cette perspective, l'attention portée aux agents de développement rural éclairerait non seulement l'émergence d'autres «métiers flous» directement comparables mais aussi de nombreuses situations dans lesquelles accorder des marges importantes d'initiative à des agents publics apparaît comme la seule solution face à certains objets ou interlocuteurs difficilement saisissables.

Bibliographie

Béhar, D., Estèbe P., 1996. Le chef de projet et le sous-préfet à la ville. Espace et sociétés, 84-85, $37-$ 43.

Baron Cécile, Bureau Marie Christine, Le Dantec Eliane, Nivolle Patrick (1994), Les intermédiaires de l'insertion, Dossier du centre d'études de l'emploi, $\mathrm{n}^{\circ} 1,71 \mathrm{p}$.

Bourdieu P. (dir.), 1993. La misère du monde, Le seuil, Paris.

Brévan C., Picard P., 2000. Une nouvelle ambition pour les villes, de nouvelles frontières pour les métiers, Rapport à Monsieur Claude Bartolone, Ministre délégué à la ville.

Burlen C., 1998. Du pouvoir de coordination des secrétaires généraux de communes. in : Balme R., Faure A., Mabileau A., Politiques locales et transformations de l'action publique en Europe, Cerat, Grenoble.

Cousin O., 1996. Les mutations du travail social : des transformations du public aux changement dans les modes de prise en charge. Sociologie du travail, (2), 141-161.

Demazière D., Mercier D., 2003, La tournée des facteurs ? Normes gestionnaires, régulations collectives et stratégies d'activité. Sociologie du travail, 45 (2), 237-258.

Donzelot J., Estèbe P., 1994. L'Etat animateur, essai sur la politique de la ville. Esprit, Paris.

Dubar C., Tripier P., 1998. Sociologie des professions. Armand colin, Paris.

Duran P., Thoenig J.-C., 1996. L'Etat et la gestion publique territoriale. Revue française de sciences politiques, 46 (4), 580-622.

Faure A., 1992. Le village et la politique. Essai sur les maires ruraux en action, Paris, l'Harmattan, Paris.

Gaudin, J.-P., 1999. Gouverner par contrat : l'action publique en question. Presses de Sciences Po, Paris.

Gerbaux F. 1998, Développement rural en Europe, politiques publiques et recomposition des territoires. in : Balme R., Faure A., Mabileau A. (eds), Politiques locales et transformations de l'action publique en Europe. Cerat, Grenoble. 
Grémion P., 1976. Le pouvoir périphérique, bureaucrates et notables dans le système politique français. Le Seuil, Paris.

Ion J., 1990, Le travail social à l'épreuve du territoire. Privat, Toulouse.

Ion J., Péroni M., Engagement public et exposition de la personne, L'aube, La tour d'aigues.

Jeannot G., 2001. Le travail public, un exemple dans le développement local. in : Jeannot G., Veltz P. (eds.), Le travail entre l'entreprise et la cité. L'Aube, La tour d'Aigues, pp. 125-132.

Jeannot G., 2001 (b). L'impossible fin de la solution équipement. Les annales des ponts et chaussées, 99, 4-14.

Lascoumes P., Le Bourhis J.-P. 1997 L'environnement ou l'administration des possibles, L'Harmattan, Paris.

Lorrain D., 1989. 570000 professionnels de l'urbain, la fonction communale aujourd'hui. Les annales de la recherche urbaine, 44-45,127-138.

Maillard J. de, 2000. Les Chefs de projet et les recompositions de l'action urbaine, un nouveau métier urbain. Les annales de la recherche urbaine, 88, 6-17.

Maillard J. de, 2002. Les travailleurs sociaux en interaction. Politiques sociales urbaines, mobilisations des professionnels et fragmentation. Sociologie du travail, 44 (2), 215-232.

Mouton Ph. 1995. Les collectivités territoriales et le développement social urbain : la transition du Xe au XIe Plan, Etudes et réflexions CNFPT, $\mathrm{n}^{\circ} 18$, octobre.

Nay O., Smith A., 2002, Le gouvernement du compromis, Courtiers et généralistes dans l'action publique. Economica, Paris.

Osty F., 2003. Le désir de métier, engagement, identité et reconnaissance au travail. PUR, Rennes.

Pallez F., Aggeri F., 2002. Les nouvelles figures de l'Etat dans les mutations industrielles. Cahiers du CGS, 20.

Péraldi M., 1995. Le cycle du fusible. Jalons pour une histoire sociale du DSU à Marseille. Les annales de la recherche urbaine, $\mathrm{n}^{\circ}$ 68-69, 69-73.

Piotet F. (dir.), 2002, La révolution des métiers. PUF, Paris.

Roch M.-F., 1983. De PAR en PAR ou réflexions théoriques sur la pratique professionnelle d'un aménageur en zone rurale. Doctorat de troisième cycle UER urbanisation, aménagement, Université des sciences sociales de Grenoble.

Sewell, W.-H., 1983. Gens de métiers et révolutions : le langage du travail de l'Ancien Régime à 1848, Aubier, Paris.

Sipp J.-F, Blanc M., 2000. Les métiers de la ville et du développement social urbain, rapport pour la délégation interministérielle à la ville

Tapie-Grime M., 1993. Structuration professionnelle du développement local. Territoire, juin, 27-30.

Terssac G. de, 2003. Vers une sociologie des activités professionnelles. Communication aux Journées de sociologie du travail, Paris.

Vaivre F., 2001. Les pays dans la dynamique intercommunale, Analyse des jeux d'acteurs et des modes de construction territoriale. Thèse de doctorat, Université de Franche Comté.

Wuhl S. 1996, Insertion : les politiques en crise. PUF, Paris.

Résumé : avec la notion de développement sont apparus des «nouveaux métiers » qui se caractérisent par une prescription globale très vague, une ambigü̈té des rôles en particulier en relation avec les élus locaux, des statuts bricolés et des parcours irréductibles aux carrières de fonctionnaires. A partir d'un suivi des agents de développement rural sur trois décennies, l'article met en évidence que, si l'autonomie de ces agents est d'abord liée à une phase d'invention liée au glissement de l'aménagement au développement et de recomposition des partages entre sphère publique et privée, l'ambiguïté des rôles perdure et se diffuse jusqu'aux parcours professionnels du fait de conditions sociales de l'emploi particulières : en l'occurrence, dans un contexte de co-financement de l'action publique territoriale, la formation d'un couple entre un élu désireux d'investir un territoire intercommunal et un agent de développement qui se "créée son poste» en captant diverses subventions. Pour cette période se dégage alors une figure singulière de "métiers flous ». 